\title{
Pediatric Palliative Care at the Children's Cancer Center of Lebanon: 2 Case Reports
}

\author{
Miguel R. Abboud Manal Azzi Samar Muwakkit \\ Children's Cancer Center of Lebanon, American University of Beirut Medical Center, Beirut, Lebanon
}

\section{Introduction}

Sustained effort has been focused on pediatric palliative care in developed countries. Reports on this topic produced by the Institute of Medicine of The National Academy of Science in the United States and the World Health Organization identified significant areas where improvement was needed, in particular the need for more research and the development of new models of care [1-3].

Health care workers in tertiary care centers play a key role in the successful management of childhood cancer. However, in the best of circumstances, about $25 \%$ of children diagnosed with cancer eventually die of their disease. Therefore, care providers need to be geared towards the total care of patients who are not responsive to curative treatment and ensure control of pain and other symptoms, as well as psychological, social, and spiritual support.

The end-of life care service acquires an added dimension of complexity in countries where resources are limited and it competes with curative care for cancer patients. This is further compounded by the absence of community-based organizations that complement the tertiary center in providing adequate end-of-life care, thus sparing resources required for other vital medical services. An additional obstacle to optimal palliative care is that in many countries there is an absence of a legal framework that supports parent, patient and physician decision making at the end of life [4].

\section{KARGER}

Fax +4161306 1234

E-Mail karger@karger.ch

www.karger.com
(C) 2007 S. Karger AG, Basel

1011-7571/07/0167-0050\$23.50/0

Accessible online at:

www.karger.com/mpp
The Children's Cancer Center of Lebanon at the American University of Beirut Medical Center provides care to about 100 new patients with cancer a year and a large number of children with hematological disorders. All newly diagnosed cases are treated using modern protocol-based therapy and only a few patients previously treated elsewhere are accepted for care. A multidisciplinary team approach for handling end-of-life care, relying primarily on the tertiary care center staff, volunteers and facilities, has been developed. The two cases presented here reflect some of the issues that are at the core of a palliative care service for children with cancer, particularly those with poor prognosis.

\section{Case Report 1}

Zein, a 4-year-old boy, was diagnosed with metastatic neuroblastoma. Certain features of his disease were associated with a high risk for recurrence, and this possibility was discussed with his family early on. He underwent high-dose chemotherapy and hematopoietic stem cell transplant successfully, but was found to have a bone marrow recurrence 6 months later. At that time, the attending physician met with the parents, together with a psychologist, primary nurse and one of the volunteers at the center to whom the family was close. After they were informed of the results, the option of a second-line therapy was discussed and the family opted for no therapy, as he was symptom free and the parents believed that there was no real possibility of a cure for their child. Two months later, he developed pain and was started on oral morphine sulfate and then fentanyl patches. As the pain 
worsened and he developed raccoon eyes, he was hospitalized for intravenous morphine. At this time, a second meeting was held with the family, during which they asked that a central line be placed and that palliative chemotherapy be started after they reviewed all potential toxicities. He received 1 cycle of chemotherapy and had a prompt response of bony pain and a clearing of the discoloration around his eyes. He then went on 4 more cycles of chemotherapy as an outpatient and remained pain free for 5 months with no significant toxicity due to chemotherapy and no hospitalizations. At the time of this writing, he is still alive and active, but plans have been made with the family to face the eventual disease recurrence.

\section{Case Report 2}

Reem was a 14-year-old girl with osteogenic sarcoma of the right distal femur. She received chemotherapy and underwent limb salvage surgery. At the time of surgery, a poor tumor response to previous chemotherapy was noticed, which is an adverse prognostic factor. The patient and family were informed of this, and arrangements were made to add new chemotherapeutic agents to her regimen. Six months after the end of chemotherapy, she was found to have a mestastatic solitary lung nodule. This was surgically removed, but 3 months later, 2 more nodules were detected, which were also surgically removed and salvage chemotherapy was started. After 3 cycles of chemotherapy, she was found to have multiple lung nodules and a large unresectable mass. After discussions with the patient and family, a decision was made to stop chemotherapy and care for the patient at home. She became progressively dyspneic, and therefore oxygen was started at home. The parents were advised that they could come to the hospital at any time should the need arise or if they felt uncomfortable at home. Oral morphine and later fentanyl patches were also started. The patient complained of fatigue, shortness of breath and pain. Home visits were made by one of the center's physicians, and she continued to come to the center to attend special school classes and to receive blood transfusions. Six days before her death, the family called and said that they could not cope with the situation at home because of the increasing pain and dyspnea. She was admitted to the hospital and started on intravenous morphine as needed every $3 \mathrm{~h}$. The house staff was concerned about using high doses of opioid for several reasons. After discussions with the house staff and family, the patient was started on a continuous morphine drip. An initial complete blood count and an electrolyte panel were obtained as well as a chest CT to look for pneumonia or a pleural effusion. No further laboratory studies were obtained. Her pain and dyspnea were controlled and she died peacefully with her family beside her 5 days later.

\section{Discussion}

Palliative care should be an integral part of the care plan for every patient, with different degrees of emphasis according to the clinical picture. Early and active inter- vention is necessary for patients with diseases that have a high risk of treatment failure, such as brain stem gliomas and neuroblastoma. Early recognition of treatment failure and frank discussions of available options with the family, as happened in Zein's case, facilitate better planning, integration of palliative care and allow more time for families to adjust $[5,6]$. When his cancer relapsed, the blending of care at home by the parents with that of the hospital facility made it possible for Zein to achieve two main goals of end-of-life therapy - extension of life and maximizing its quality. These have been demonstrated to be clearly recognized goals of parents for their children at the end of life [5].

Case No. 2 again illustrates the complementary roles of different components of the provision of end-of-life care. Reem's lack of response to treatment was addressed jointly with the patient and her family. The decision was to stop chemotherapy and care for her at home. That was underpinned by Reem's access to the care center's physicians through home visits, special school and clinical support facilities and at the end to management of pain and other symptoms at the hospital.

Pain management was a significant problem in this patient. There are several obstacles to pain management at the end of life. Reem was initially well managed with round-the-clock oral morphine and later fentanyl patches. Upon admission to the hospital, she was seen by the general pediatric team and morphine as needed was started. This way of administering morphine or other opioids is ineffective in this setting. Underdosing of opioids is often the result of ignorance of pain management protocols and the irrational fear of addiction among practitioners. An additional obstacle to pain management at the end of life is the fear of many practitioners that opioids may hasten the death of patients, even though there is little evidence that appropriate opioid use at the end of life hastens death $[1,7,8]$. These issues highlight the need for special training of caregivers in the use of medications for pain relief at the end of life $[5,7,9]$. Also important is the development of a legal framework that will legalize the current practice in many centers in Lebanon [4].

Two other important issues are raised in this case: the first is the issue of daily activities and schooling and the second is the location of care for a dying patient. Quality of life is very important for children and their families at the end of life. In Reem's case, the medical support she received, for example transfusions, giving her the ability to attend school and conduct other daily activities greatly enhanced her quality of life. In the United States, about 
half the children with cancer die at home [8]. Management of the dying child may be carried out at home or in the hospital, but significant resources are needed for offering the choice of home or hospital care at the end of life. Hospice care is not available in many parts of the developing world. In Lebanon, hospice would be a welcome addition and complement care offered at our center and other pediatric oncology programs. This is important for the optimization of care and better utilization of resources. Significant involvement of health care practitioners and the community as well as the development of a legal framework governing the delivery of care at the end of life are required. These and other issues have been addressed in two recent reviews $[5,10]$.

\section{Conclusion}

These two case reports show how a combination of home and hospital care can be utilized in the provision of palliative care for a dying child based on his or her needs as agreed upon by the family and the professional caregivers.

\section{Acknowledgements}

We thank Mrs. Christiane Makarem, Mr. Hassan Daouk, and CCCL volunteers at the Children's Cancer Center of Lebanon for their efforts and continuous help.

\section{References}

1 Field MJ, Behrman RE, Committee on Palliative and End-of-Life Care for Children and Their Families: When Children Die: Improving Palliative and End-of-Life Care for Children and Their Families. Washington, National Academy Press, 2003.

2 World Health Organization: Cancer Pain Relief and Palliative Care in Children. Geneva, World Health Organization, 1998.

3 World Health Organization: Cancer Pain Relief and Palliative Care. Report of a WHO Expert Committee. Geneva, World Health Organization, 1990.

4 The Lebanese Order of Physicians in Tripoli in Collaboration with the Lebanese Cancer Society (Palliative Group) and The Lebanese National Consultative Ethics Committee: Conference on palliative and supportive care for cancer patients. Tripoli, March 2007.
5 Wolfe J, Sourkes B: Palliative care for the child with advanced cancer; in Pizzo PA, Poplack DG (eds): Principles and Practice of Pediatric Oncology: Palliative Care for the Child with Advanced Cancer, ed 5. Philadelphia, Lippincott Williams \& Wilkins, 2006.

6 Wolfe J, Klar N, Grier HE, Duncan J, SalemSchatz S, Emanuel EJ, Weeks JC: Understanding of prognosis among parents of children who died of cancer: impact on treatment goals and integration of palliative care. JAMA 2000:284:2469-2475.
7 Abrahm JL: A Physician's Guide to Pain and Symptom Management in Cancer Patients. Baltimore, The Johns Hopkins University Press, 2000.

8 Wolfe J, Grier HE, Klar N, Levin SB, Ellenbogen JM, Salem-Schatz S, Emanuel EJ, Weeks JC: Symptoms and suffering at the end of life in children with cancer. $\mathrm{N}$ Engl J Med 2000;342:326-333.

9 American Pain Society: Principles of Analgesic Use in the Treatment of Acute Pain and Cancer Pain, ed 4. Glenview, American Pain Society, 1999.

10 Hurwitz CA, Duncan J, Wolfe J: Caring for the child with cancer at the close of life: 'there are people who make it, and I'm hoping I'm one of them'. JAMA 2004;292:21412149. 